\title{
Murine pituitary tumor-transforming gene functions as a securin protein in insulin-secreting cells
}

\author{
Run Yu, Martha Cruz-Soto, Sergio Li Calzi, Hongxiang Hui and Shlomo Melmed \\ UCLA School of Medicine, Cedars-Sinai Research Institute, Cedars-Sinai Medical Center, 8700 Beverly Blvd, Room D3066, Los Angeles, California 90048, USA \\ (Requests for offprints should be addressed to R Yu; Email: run.yu@cshs.org)
}

\begin{abstract}
Human pituitary tumor-transforming gene 1 (PTTG1) encodes a securin protein critically important in regulating chromosome separation. Murine PTTG (mPTTG) is 66\% homologous to human PTTG1 and PTTG-null (PTTG-/ -) mice exhibit pancreatic $\beta$-cell hypoplasia and abnormal nuclear morphology with resultant diabetes. As we show that ductal $\beta$-cell neogenesis is intact in PTTG $-/-$ mice, we explored mechanism for defective $\beta$-cell replication. We tested whether mPTTG exhibits securin properties in mouse insulin-secreting insulinoma MIN6 cells, using a live-cell system to monitor mitosis in cells transfected with an enhanced green fluorescent protein (EGFP)-tagged mPTTG conjugate (mPTTG-EGFP). To fulfill the criteria for securin properties, the protein should undergo degradation immediately before the metaphaseto-anaphase transition when expression levels are low, and should inhibit metaphase-to-anaphase transition when
\end{abstract}

expression levels are high. EGFP itself did not undergo degradation throughout mitosis and high levels of EGFP per se did not affect normal mitosis progression $(n=25)$. However, mPTTG-EGFP was degraded 2 min before the metaphaseto-anaphase transition when expression levels were low $(n=19)$, and high mPTTG-EGFP levels blocked metaphaseto-anaphase transition in 13 cells. mPTTG-EGFP inhibited MIN6 cell proliferation and caused apoptosis. Immunocoprecipitation demonstrated binding of mPTTG-EGFP and separase. These results show that $\mathrm{MPTTG}$ exhibits properties consistent with a murine securin in insulin-secreting mouse cells and mPTTG overexpression inhibits cell proliferation, suggesting that defective $\beta$-cell proliferation observed in PTTG $-/-$ mice is likely due to abnormal cell-cycle progression.

Journal of Endocrinology (2006) 191, 45-53

\section{Introduction}

Pituitary tumor-transforming gene 1 (PTTG1) is an oncogene that is overexpressed in pituitary tumors and other neoplasms (Zhang et al. 1999, Yu \& Melmed 2001, 2004). Human PTTG1 is identified as securin, a protein regulating chromosome separation (Zou et al. 1999, Nasmyth 2002, Yu et al. 2003). Securin inhibits separase, which degrades the bonding proteins between sister chromatids at the metaphaseto-anaphase transition. Securin is proteolyzed by the anaphasepromoting complex after chromosomes are aligned at the equatorial plate and the mitotic checkpoint satisfied (Nasmyth 2002). Securin, therefore, appears to prevent premature chromosome separation (Nasmyth 2002). In live human cells, enhanced green fluorescent protein (EGFP)-tagged human PTTG1 is degraded 1-2 min before sister chromatid separation; whereas high levels of EGFP-tagged human PTTG1 prolong metaphase and inhibit chromosome separation, resulting in aneuploidy (Yu et al. 2003).

Murine PTTG (mPTTG) amino acid sequence is $66 \%$ homologous to human PTTG1 (Wang \& Melmed 2000). PTTG-null (PTTG-/-) mice are viable but exhibit a variety of abnormalities, including splenic hypoplasia, thymic hyperplasia, small testes, thrombocytopenia, and sexually dimorphic diabetes mellitus (Wang et al. 2001, 2003). These mice also develop organ-specific abnormal cell nuclear morphology, such as macronuclei in the pancreatic $\beta$-cells and the hepatocytes (Wang et al. 2003, Akino et al. 2005). Pancreatic $\beta$-cell proliferation is slower and islet size is much smaller than wild-type (WT) in both male and female PTTG - / - mice, but only male mice develop overt diabetes apparently due to lower insulin sensitivity (Wang et al. 2003, Fraenkel et al. 2006). $\beta$-Cell apoptosis appears normal in PTTG - / - mice (Wang et al. 2003). Similar defects in $\beta$-cell proliferation occur in mice deficient in cyclin D (Georgia \& Bhushan 2004, Kushner et al. 2005) or cyclin-dependent kinase (CDK) 4 (Rane et al. 1999), and cyclin D1 overexpression causes $\beta$-cell hyperplasia (Zhang et al. 2005), suggesting that mPTTG may function as a cell-cycleregulating protein in pancreatic $\beta$-cells.

As mPTTG shares high homology with human PTTG1 and PTTG - / - mice exhibit abnormal nuclear morphology in the $\beta$-cells, we hypothesize that mPTTG functions as a securin in insulin-secreting cells. We tested the securin function of mPTTG by live-cell imaging in insulin-secreting MIN6 mouse insulinoma cells and by immunocoprecipitation with 
separase. We also tested the effects of PTTG overexpression on proliferation of these cells. The results indicate that $\mathrm{mPTTG}$ exhibits properties consistent with those of a securin and regulates $\beta$-cell mass, thus providing a potential mechanism for defective $\beta$-cell proliferation in PTTG $-/-$ mice.

\section{Materials and Methods}

\section{Cells and plasmids}

Mouse MIN6 insulinoma cells (passages 26-34) and human H1299 cells were grown in Dulbecco's modified Eagle's medium (DMEM) with 10\% fetal bovine serum (FBS) at $37^{\circ} \mathrm{C}$ with $5 \% \mathrm{CO}_{2}$. Mouse PTTG-EGFP was constructed by cloning mouse PTTG into pEGFP-N3 (Clontech) using EcoR1 and BamH1 restriction sites with EGFP at the C terminus of PTTG, and the sequence verified by DNA sequencing. MIN6 cells were transfected using Lipofectamine PLUS and H1299 cells using Lipofectamine 2000 (Invitrogen). Transfection was carried out as recommended by the manufacturer. One day after transfection, MIN6 cells were split onto coverslips coated with cell adhesive ECL (Upstate, Charlottesville, VA, USA) or regular dishes and cells studied (microscopy or western blot) 2 days after transfection.

\section{Animals}

WTand PTTG - / - mice (Wang et al. 2001, 2003) between 3 weeks and 8 months of age were kept in a hybrid background derived from C57/BL6 and 129SvJ mouse strains. Mice were housed in a $12 \mathrm{~h}$ light: $12 \mathrm{~h}$ darkness cycle and fed standard chow ad libitum. WTand PTTG-/ - male mice were killed at 3 weeks or 8 months (three mice per group) and pancreata harvested and fixed in 10\% formalin and embedded in paraffin.

\section{Immunocoprecipitation and western blot}

Semi-confluent human H1299 cells were cotransfected with plasmids encoding V5 epitope-tagged separase (from Dr DeCaprio, Dana Farber Cancer Center, Boston; Chestukhin \& DeCaprio 2003) and mPTTG-EGFP overnight. Experiments were then carried out at $4{ }^{\circ} \mathrm{C}$ or on ice. Cells were lysed with lysis buffer $(\mathrm{NaCl} 140 \mathrm{mM}$, Tris-Cl $50 \mathrm{mM}$, and NP-40 0.5\% (pH $7 \cdot 6)$ ), plus protease inhibitor cocktail (Roche) for $30 \mathrm{~min}(1 \mathrm{ml}$ lysis buffer per $10 \mathrm{~cm}$ dish). Ten microliters of lysate were mixed with equal amount of $2 \times$ SDS sample buffer and run on SDSPAGE and the remainder spun for $20 \mathrm{~min}$. One milliliter of supernate was either incubated with rabbit polyclonal anti-EGFP or normal rabbit serum (both 1:200), or with mouse monoclonal anti-V5 or anti-FLAG (both $2 \mu \mathrm{g} / \mathrm{ml}$ at final concentration) for 3 h. Protein A/G PLUS agarose (Santa Cruz Biotechnology, Santa Cruz, CA, USA) was added and incubation extended for another $90 \mathrm{~min}$. The agarose beads were washed and bound proteins dissociated with $2 \times$ SDS sample buffer, resolved on SDS-PAGE, and transferred to polyvinylidene difluoride (PVDF) membrane. For western blots, the membrane was blocked with $5 \%$ non-fat dry milk in PBS and mouse monoclonal anti-V5 and anti-EGFP were used at 1:2000, and rabbit polyclonal anti-EGFP at 1:1000, followed by horseradish peroxidase-labeled secondary antibodies. The membrane was visualized with enhanced chemoluminescent reagent.

\section{Immunofluorescent staining}

MIN6 cells transfected with mPTTG-EGFP were fixed in 4\% paraformaldehyde in PBS, permeabilized with $0.6 \%$ Tween-20 in blocking buffer, and stained with Hoechst 33342 (1:5000). For insulin staining in MIN6 cells plated on coverslips, a guinea pig insulin antibody (Dako, Carpinteria, CA, USA) was used at 1:100 dilution, followed by a rhodamine-labeled anti-guinea pig secondary antibody. For immunofluorescent staining of pancreatic sections, they were deparaffinized, rehydrated, permeabilized, and incubated with guinea pig anti-insulin at 1:50 dilution and rabbit anti-PDX-1 at 1:2000 dilution (from Dr Michael German, University of California, San Francisco, USA), followed with rhodaminelabeled anti-guinea pig IgG and fluoresceine-labeled antirabbit IgG, and counterstained with diamidinophenylindole (DAPI) for DNA labeling. Sections were examined using a Nikon Eclipse TE200 fluorescence microscope. Pictures were captured using a SPOT digital camera (Diagnostic Instruments, Inc., Sterling Heights, MI, USA) and processed with Adobe Photoshop.

Single, live-cell imaging has been described in detail ( $\mathrm{Yu}$ et al. 2003, Boelaert et al. 2004). Cells were perfused with $\mathrm{CO}_{2^{-}}$ independent L15 medium (Invitrogen) supplemented with 10\% FBS and saturated with ambient air in an FCS-2 closed perfusion system (Bioptechs, Butler, PA, USA) at $37^{\circ} \mathrm{C}$. The perfusion chamber was placed on the stage of a Nikon inverted fluorescence microscope and the cells were observed with a $40 \times$ extra-long working distance objective. Duration of mitosis was determined by counting the minutes between prophase and telophase. Both phase-contrast and EGFP-fluorescent images were acquired with a CCD SPOT digital camera. Fluorescence intensity was objectively determined using two neutral density filters (NDFs). Each NDF reduces incident light intensity by $50 \%$. High fluorescence was assigned to a cell if the cell is clearly visualized after application of two NDFs, medium fluorescence assigned if the cell is clearly visualized after application of one (but not two) NDF(s), and low fluorescence assigned if the cell is only visualized when neither NDF was applied.

\section{Results}

Since both replication and neogenesis contribute to $\beta$-cell mass expansion (Bonner-Weir 2000) and PTTG deletion causes defective $\beta$-cell proliferation, we first tested whether $\beta$-cell neogenesis required PTTG. Pancreatic ducts were examined for signs of islet neogenesis in young and old mice by staining for insulin and transcription factor pancreatic duodenal homeobox 1 


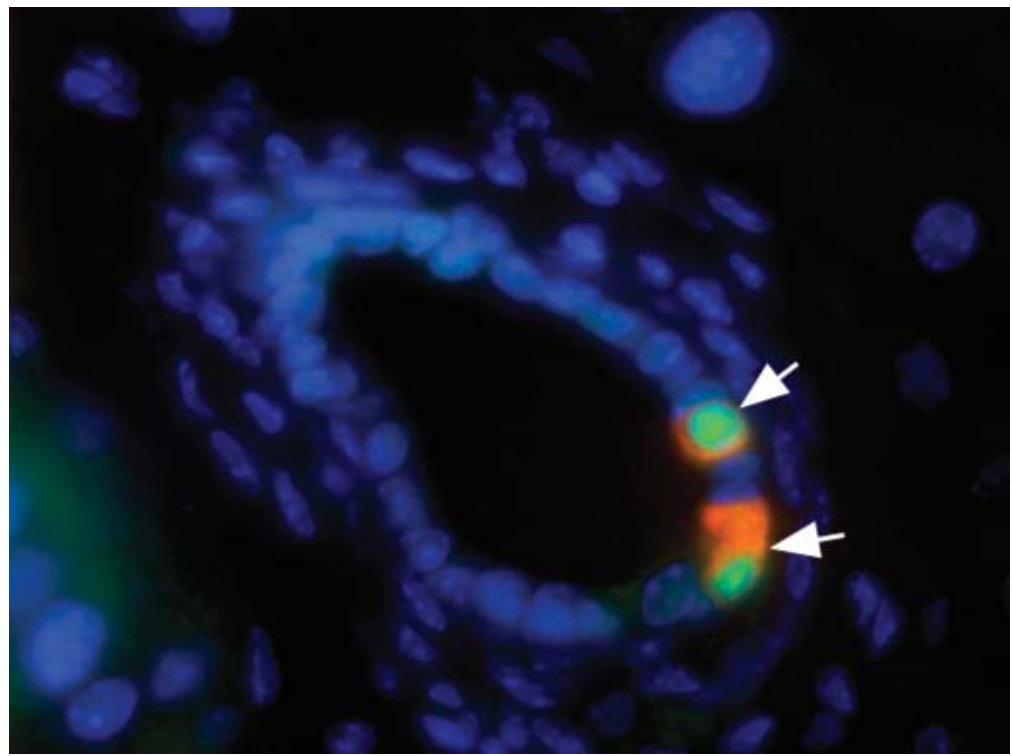

Figure 1 Pancreatic $\beta$-cell neogenesis in 3 -week-old mice. Paraffin sections of pancreata from 3-week-old PTTG-null mice were stained with guinea pig anti-insulin (red) and rabbit anti-PDX-1 (green), followed with rhodamine-labeled anti-guinea pig $\operatorname{lgG}$ and fluoresceine-labeled anti-rabbit IgG. Nuclei were counterstained with DAPI (blue). A ductal structure with two cells positive for insulin and PDX-1 (arrows) is shown.

(PDX-1), both postnatal markers of $\beta$-cells (Ashizawa et al. 2004). In 3-week-old PTTG-/ - mice, insulin and (PDX-1) immunoreactive cells lining pancreatic ducts were detected (Fig. 1) in 5 out of 100 ductal structures examined. In contrast, neogenesis was not observed in 100 ductule structures in 3 -week-old WT male mice $(P=0.03$ by Fisher's exact test). Neogenesis was not seen in 8-month-old hyperglycemic PTTG - / - or WT male mice (100 ductal structures observed per group). These results indicate that defective $\beta$-cell replication rather than differentiation was likely the major cause of diminished $\beta$-cell mass in PTTG-null mice.

To study the mechanisms of defective $\beta$-cell replication after PTTG deletion, we next tested the hypothesis that murine PTTG (mPTTG) fulfills the properties of a securin protein. An EGFP-tagged mPTTG (mPTTG-EGFP) was constructed for live-cell imaging. Western blot demonstrates that MIN6 cells transfected with mPTTG-EGFP expressed predominantly 55 kDa mPTTG-EGFP with minimal levels of EGFP (Fig. 2);
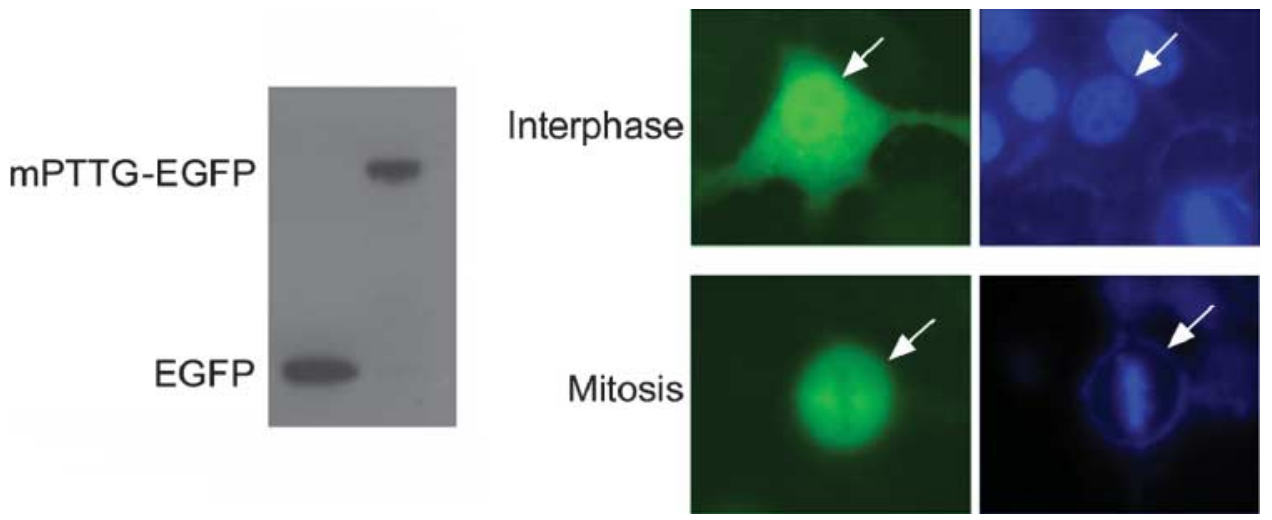

Figure 2 Expression of mPTTG-EGFP in MIN6 cells. Left; Western blot of lysates from MIN6 cell transfected with EGFP or mPTTG-EGFP. MIN6 cells were transfected with plasmids encoding EGFP alone (left lane) or mPTTG-EGFP (right lane). Cells were lysed 2 days after transfection and subjected to western blot using a monoclonal anti-EGFP antibody. Right; intracellular localization of mPTTG-EGFP during interphase and mitosis. MIN6 cells were transfected with mPTTG-EGFP. Cells were fixed 2 days after transfection and cell nuclei stained with Hoechst 33342 (blue) to determine the cell-cycle phase. mPTTG-EGFP fluorescence is preserved and stable throughout staining (green). mPTTG-EGFP-expressing cells are shown (arrows). 
thus, the EGFP fluorescence observed in microscopy faithfully represents $\mathrm{mPTTG}$-EGFP. In our hands, we cannot consistently quantify endogenous mPTTG protein by western blot, but the expression levels of MPTTG-EGFP in individual cells are probably higher than the endogenous PTTG, as the mPTTG-EGFP expression was driven by a strong cytomegalovirus (CMV) promoter. We next examined the intracellular localization of mPTTG-EGFP. In interphase MIN6 cells, mPTTG-EGFP was localized to both the nuclei and the cytoplasm. At metaphase, no discernable nucleus can be seen as the nuclear envelope is dissolved, and mPTTG-EGFP localized to the cytoplasm (Fig. 2), similar to human PTTG1.

We utilized a live-cell observation system (Yu et al. 2003, Boelaert et al. 2004) to study mitosis of MIN6 cells transiently expressing EGFP or mPTTG-EGFP. MIN6 cells expressing either low or high EGFP levels exhibited normal chromosomal separation and cytokinesis $(n=25$; Fig. 3$)$, indistinguishable from those of untransfected cells. EGFP fluorescence was stable throughout mitosis and equally distributed into the daughter cells, indicating stable EGFP protein levels. Mitosis duration from prophase to telophase was similar in cells expressing EGFP alone $(10.0 \mathrm{~min})$ and in untransfected cells (12.9 min; Table 1). EGFP itself, regardless of expression levels, therefore did not appear to affect mitosis or be affected by mitosis. However, mPTTG-EGFP expression dramatically prolongs mitosis duration. In cells expressing low mPTTG-EGFP levels, mitosis duration doubled compared with cells expressing EGFP alone or untransfected cells (Fig. 4, Table 1). Mitosis duration was prolonged four- to fivefold in cells expressing medium mPTTG-EGFP levels, one (out of 11) such cell died before completion of mitosis. In cells expressing high mPTTG-EGFP levels, mitosis was blocked for at least $2 \mathrm{~h}$ (Fig. 4, Table 1) in most cells, three (out of 13) cells died before mitosis duration reached $2 \mathrm{~h}$. In all cells expressing mPTTG-EGFP, green fluorescence decreased over time

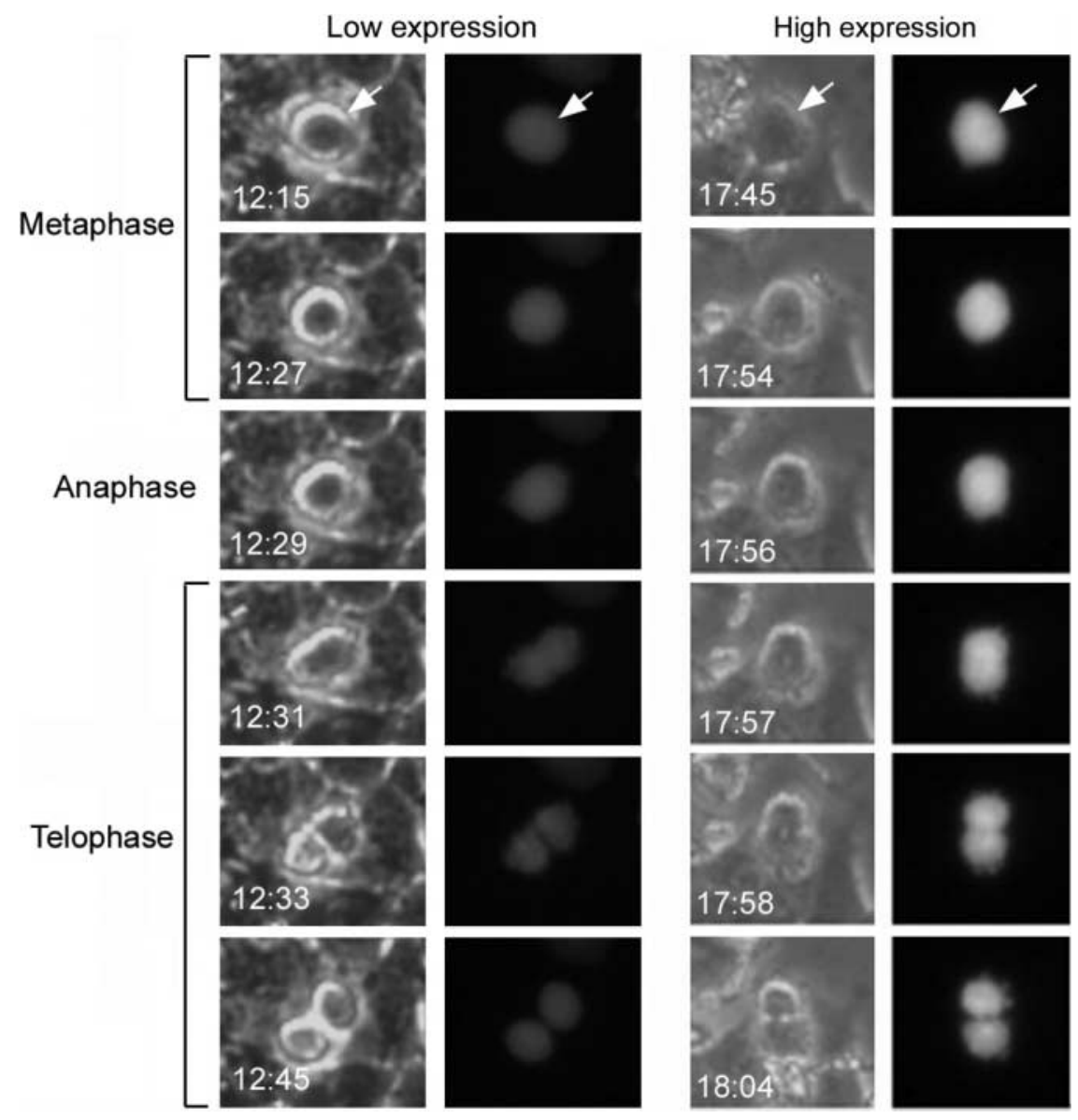

Figure 3 Live-cell imaging of MIN6 cells expressing EGFP. MIN6 cells were transfected with EGFP and live MIN6 cells were continuously observed with fluorescence microscopy and representative images shown. Phase-contrast (bright-field) and EGFP-fluorescent (green) images were taken simultaneously at the indicated times shown at the lower left corner of the phasecontrast image panel. Left; a MIN6 cell expressing low expression levels of EGFP (arrow). Right; a MIN6 cell expressing high levels of EGFP (arrow). Phases in mitosis are shown on the left of images and the labeling applies to both 'low expression' and 'high expression'. See Materials and Methods for definitions of low or high expression levels. 
Table 1 Mitosis duration in MIN6 cells transfected with EGFP or mouse PTTG-EGFP

\begin{tabular}{|c|c|c|c|c|c|}
\hline & \multirow[t]{2}{*}{ Untransfected } & \multirow[t]{2}{*}{ EGFP } & \multicolumn{3}{|l|}{ PTTG-EGFP } \\
\hline & & & Low & Medium & High \\
\hline Cells $(n)$ & 15 & 15 & 15 & 8 & 11 \\
\hline Duration (min) mean \pm s.D. & $12 \cdot 9 \pm 1 \cdot 6$ & $10 \cdot 0 \pm 1 \cdot 1$ & $24 \cdot 1 \pm 4 \cdot 3$ & $50 \cdot 6 \pm 3 \cdot 4$ & All $>120$ \\
\hline
\end{tabular}

Live untransfected cells and cells transfected with EGFP or mouse PTTG-EGFP were observed throughout mitosis. Because MIN6 cells are small and chromosomes are clearly visualized in only some cells, individual subphases of mitosis could not be reliably distinguished. Rather, the duration from prophase to telophase was measured. Cells expressing PTTG-EGFP were subdivided into three categories according to expression levels (see Materials and Methods). When the duration is longer than $2 \mathrm{~h}$, observation was stopped and the duration determined as '>120 min'.

(Fig. 4), indicating mPTTG-EGFP degradation. Only in cells expressing low or medium levels of mPTTG-EGFP, the degradation was complete at about $2 \mathrm{~min}$ before anaphase. None of the cells expressing mPTTG-EGFP $(n=43)$ underwent division without complete mPTTG-EGFP degradation. These results are consistent with a securin function of $\mathrm{mPTTG}$.

As cyclin D1 deletion results in defective $\beta$-cell replication (Kushner et al. 2005) and its overexpression causes $\beta$-cell
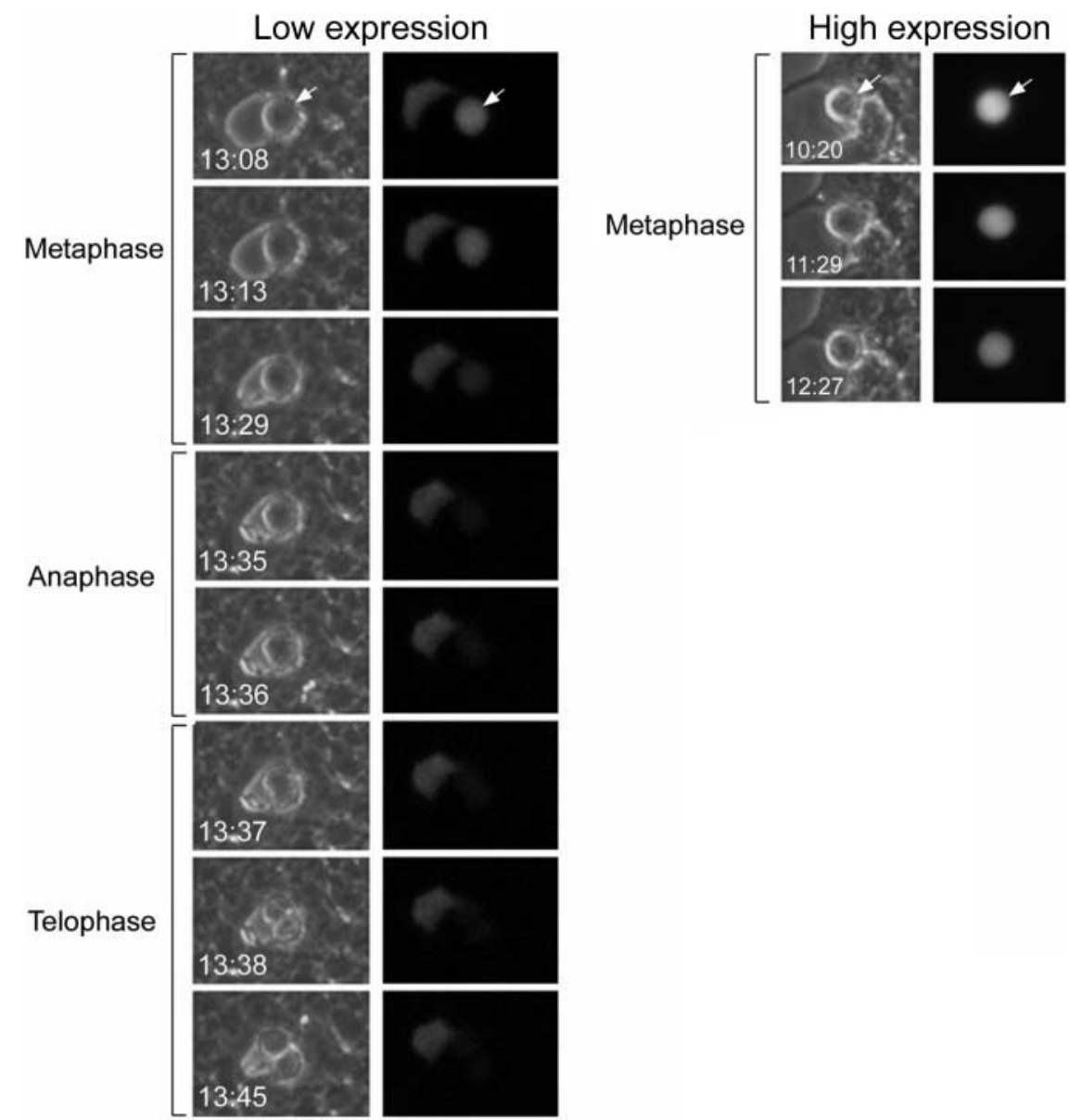

Figure 4 Live-cell imaging of MIN6 cells expressing mPTTG-EGFP. MIN6 cells were transfected with mPTTG-EGFP and live MIN6 cells were continuously observed with fluorescence microscopy and representative images shown. Phase-contrast (bright-field) and mPTTG-EGFPfluorescent (green) images were taken simultaneously at the indicated times shown at the lower left corner of the phase-contrast image panel. Left; a MIN6 cell expressing low expression levels of mPTTG-EGFP (arrow). Right; a MIN6 cell expressing high levels of mPTTG-EGFP (arrow). Phases in mitosis are shown on the left of images. See Materials and Methods for definitions of low or high expression levels. 


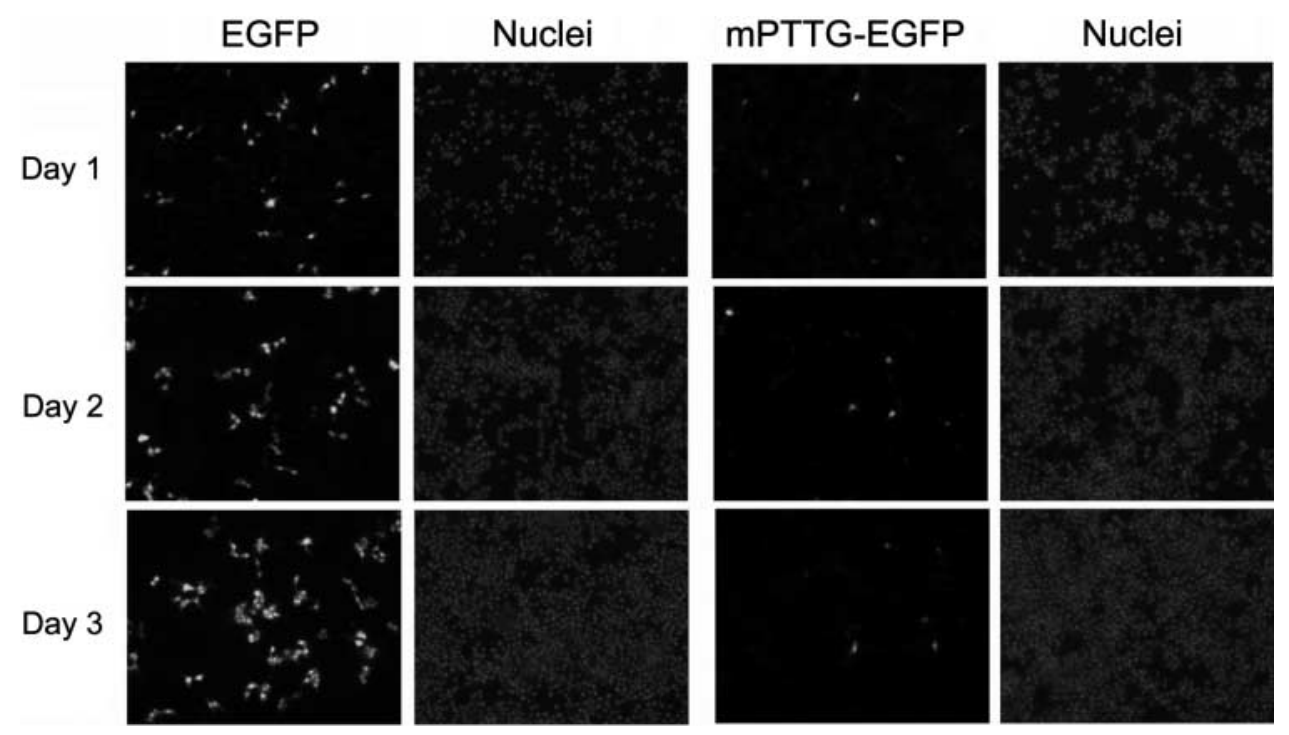

Figure 5 mPTTG-EGFP expression and MIN6 cell proliferation. MIN6 cells were transfected with EGFP alone (left) or mPTTG-EGFP (right). Cells in separate dishes were fixed with paraformaldehyde 1, 2, or 3 days after transfection, permeabilized, and nuclei stained with Hoechst 33342 (blue). EGFP and mPTTG-EGFP fluorescence is preserved and stable throughout staining (green). Representative fields are shown.

hyperplasia (Zhang et al. 2005), and PTTG deficiency causes similar defective $\beta$-cell replication, PTTG overexpression may affect cell replication. We, therefore, tested the effects of mPTTG-EGFP expression on MIN6 cell replication. MIN6 cells transfected with EGFP proliferated similarly to nontransfected cells (Fig. 5): the number of EGFP-positive cells per low-power field on D1, D2, and D3 was $35 \pm 2,81 \pm 3$, and $147 \pm 6$, while that of EGFP-negative cells was $378 \pm 14$, $1082 \pm 46$, and $1835 \pm 70$ respectively. On any given day, the percentage of EGFP-positive cells was about 8\%. Division of cells expressing EGFP was evident as shown by the clusters of EGFP-positive cells 2 or 3 days after transfection. However, MIN6 cells expressing mPTTG-EGFP did not appear to proliferate, as the number of green cells did not increase, green cells remain solitary, and no clusters of green cells were observed. Apoptosis of MIN6 cells was studied by examining nuclear morphology with fluorescence microscopy. Condensed, fragmented apoptotic nuclei were seen in cells expressing MPTTGEGFP (Fig. 6), while EGFP alone did not cause significant apoptosis. mPTTG-EGFP-induced apoptosis became more severe over time and $18 \%$ cells expressing mPTTG-EGFP were apoptotic $72 \mathrm{~h}$ after transfection (Fig. 6).

We are interested in the effects of mPTTG-EGFP expression on insulin production and secretion. As the percentage of MIN6 cells expressing mPTTG-EGFP was very low, we did not measure insulin levels in conditioned media, which is the sum of insulin secreted from all cells, most of which did not express mPTTG-EGFP. Instead, we examined intracellular insulin levels in cells expressing mPTTG-EGFP, which was indistinguishable from those in cells without mPTTG-EGFP (Fig. 7), suggesting mPTTGEGFP did not affect insulin production or secretion.
We tested whether mPTTG-EGFP directly binds to separase by immunocoprecipitation. As shown in Fig. 5, transfection efficiency in MIN6 cells was relatively low $(\sim 8 \%)$, and for unclear reasons, transfection efficiency of mPTTG-EGFP was even lower than that of EGFP alone. We, therefore, expressed
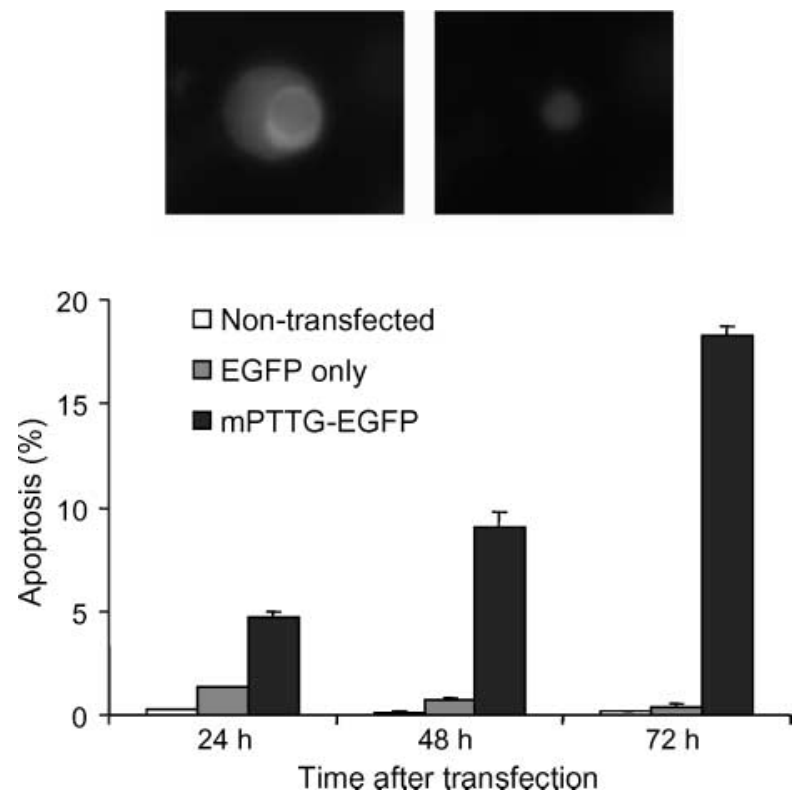

Figure 6 mPTTG-EGFP expression causes MIN6 apoptosis. MIN6 cells were transfected with EGFP alone or mPTTG-EGFP. Cells in separate dishes were fixed 24, 48, or $72 \mathrm{~h}$ after transfection and stained with Hoechst 33342. EGFP-positive cells were examined for nuclear morphology. Condensed, fragmented apoptotic nuclei were scored. Data shown are mean \pm s.D. Image: an apoptotic cell with mPTTG-EGFP. Left; mPTTG-EGFP, right; nucleus. 

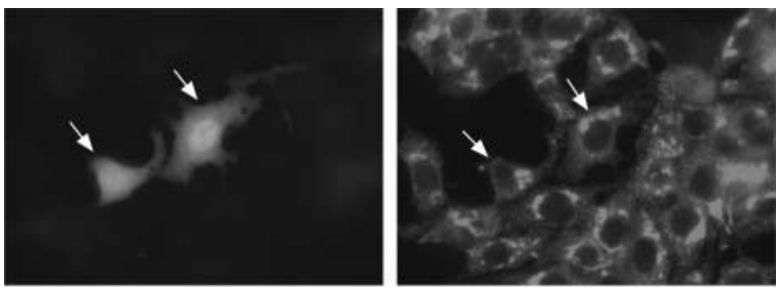

Figure 7 mPTTG-EGFP expression and insulin levels. MIN6 cells were transfected with mPTTG-EGFP. Cells were fixed 2 days after transfection and stained with a guinea pig antibody against insulin followed by rhodamine-labeled secondary antibody (red). mPTTGEGFP fluorescence is preserved and stable throughout staining (green). mPTTG-EGFP-expressing cells are marked with arrows.

mPTTG-EGFP and a V5 epitope-tagged separase (V5-separase) in H1299 cells, where transfection efficiency was higher. Expression of V5-separase or EGFP and mPTTG-EGFP was comparable within experiments (Fig. 8A). After the lysates were precipitated with anti-EGFP, anti-V5 detected V5-separase only in precipitates derived from cells expressing $\mathrm{mPTTG}$-EGFP, but not from cells expressing EGFP alone (Fig. 8B). Normal rabbit serum did not precipitate EGFP or mPTTG-EGFP, and V5-separase was not detected, as expected (Fig. 8B). These results demonstrate that intracellular mPTTG-EGFP and V5separase specifically bind to each other, while EGFP itself did not bind V5-separase. To further confirm these results, lysates were also precipitated with anti-V5 and anti-EGFP detected mPTTG-EGFP in precipitates from cells expressing $\mathrm{mPTTG}-$ EGFP but not from those expressing EGFP alone (Fig. 8C). An irrelevant anti-FLAG did not precipitate V5-separase and mPTTG-EGFP was not detected in the precipitate (Fig. 8C). The results thus indicate specific intracellular binding between mPTTG-EGFP and V5-separase.

\section{Discussion}

Although PTTG deletion results in decreased pancreatic $\beta$-cell proliferation (Wang et al. 2003), $\beta$-cell neogenesis appears unaffected as we show in this study. Mechanisms underlying defective $\beta$-cell proliferation in PTTG-null mice remain unclear, and the results shown suggest that optimal intracellular cycle-regulating protein concentration is critical for normal $\beta$-cell development.

An important clue to the pathogenesis of decreased $\beta$-cell replication is abnormal $\beta$-cell nuclear morphology, which is suggestive of abnormalities in cell-cycle regulation. mPTTG exhibits a number of functions including transcription transactivation, and upregulation of basic fibroblast growth factor in murine cells (Wang \& Melmed 2000, Ishikawa et al. 2001), but these functions are either too non-specific or cannot plausibly explain the abnormal $\beta$-cell nuclear
A

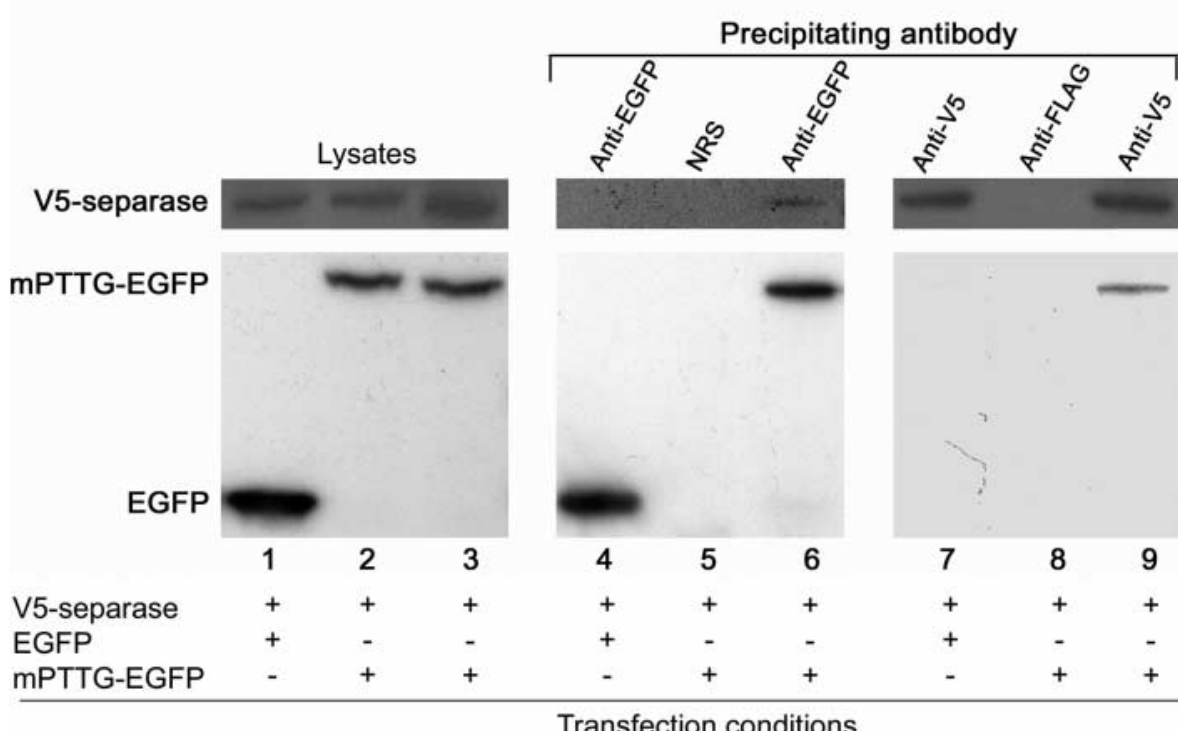

Transfection conditions

Figure 8 Immunocoprecipitation of mPTTG-EGFP and V5-separase. Lysates from $\mathrm{H} 1299$ cells cotransfected with EGFP $(1 \mu \mathrm{g}$ per $10 \mathrm{~cm}$ dish, lane 1$)$ or mouse PTTG-EGFP $(1 \mu \mathrm{g}$ per $10 \mathrm{~cm}$ dish, lanes 2 and 3$)$ and $V 5$-separase $(1 \mu \mathrm{g}$ per $10 \mathrm{~cm}$ dish) were subjected to three experiments. (A) Aliquots were subjected to SDS-PAGE, transferred, and blotted with either mouse anti-V5 (top) or rabbit anti-EGFP (bottom). (B) Lysates from cells cotransfected with EGFP or PTTG-EGFP and V5separase were immunoprecipitated with rabbit anti-EGFP (lanes 4 and 6) or normal rabbit serum (lane 5) and blotted with mouse anti-V5 (top) or mouse anti-EGFP (bottom). (C) Lysates from cells cotransfected with EGFP or PTTG-EGFP and V5-separase were immunoprecipitated with mouse antiV5 (lanes 7 and 9) or mouse anti-FLAG (lane 8) and blotted with rabbit anti-EGFP (bottom) or mouse anti-V5 (top). Transfection conditions are written under each lane. 
morphology in PTTG-null mice. As mPTTG is $66 \%$ homologous to human PTTG1 (Wang \& Melmed 2000), which is a securin in human cells, and loss of securin function may result in $\beta$-cell-cycle disruption, we tested the hypothesis that mPTTG behaves as a securin in murine insulin-secreting cells. We show by live-cell imaging that mPTTG, like human PTTG1 in human cells, functions as a securin in mouse insulinoma MIN6 cells. Based on the results of live-cell imaging of human cells expressing human PTTG, two criteria appear to be required for a protein to exhibit securin function (Yu et al. 2003, Boelaert et al. 2004): degradation immediately before metaphase-to-anaphase transition when expression levels are low and inhibition of metaphase-to-anaphase transition when expression levels are high. Both these criteria are important properties of securin. Securin degradation is the immediate step before separase starts to separate sister chromatid, while securin overexpression either directly or indirectly causes mitotic arrest (Yu et al. 2000b, 2003). Several human securin mutants only fulfill one of the criteria and thus lose proper securin function (Yu et al. 2003). Human PTTG1 fulfills these two criteria in human cells (Yu et al. 2003). mPTTG also fulfills these two criteria by virtue of degradation before cell division and inhibition of mitosis progression.

Immunocoprecipitation of mPTTG-EGFP and V5-separase demonstrates that mPTTG and separase specifically form an intracellular complex, thus lending biochemical support to the conclusion that mPTTG indeed fulfills properties of a securin. Immunocoprecipitation experiments were performed in human H1299 cells instead of in murine insulin-secreting MIN6 cells due to low transfection efficiency in the murine cells. However, since mPTTG-EGFP behaves as a securin in human H1299 cells (data not shown) and murine and human separases are highly homologous (Jager et al. 2001), it is likely that mPTTG binds to murine separase in murine cells. Another caveat of our results is that EGFP-tagged mPTTG may not fully represent wild-type mPTTG.

Deletion of several cell-cycle-regulatory proteins results in $\beta$-cell proliferation defects. Deletion of cyclins D1 and D2 or cyclin D2 alone causes defective postnatal $\beta$-cell proliferation without changes in insulin sensitivity (Georgia \& Bhushan 2004, Kushner et al. 2005), which are phenotypes similar to those observed in mice with PTTG deletion (Wang et al. 2003). Deletion of CDK4, the kinase regulated by and binding partner of cyclins D1-3, also results in defective postnatal $\beta$-cell proliferation (Rane et al. 1999). Cyclins D and CDK4 are promoters of G1- to S-phase transition; their deletion may abrogate $\beta$-cell proliferation. PTTG is thus a unique G2/M-phase gene indispensable for normal $\beta$-cell proliferation. Mice deficient in other G2/M-phase genes, such as cyclins B1 and B2 are not known for $\beta$-cell proliferation defects (Brandeis et al. 1998). Cyclin B1-null mice die in utero, and cyclin B2-null mice are apparently normal (Brandeis et al. 1998). Cyclin B2-null mice may yet develop $\beta$-cell proliferation defects in later life as overt diabetes in PTTG-null mice usually develops at 8 months, and $\beta$-cell defects were not discovered for the first reports of PTTG-null mice (Mei et al. 2001, Wang et al. 2001). Transgenic mice overexpressing cyclin D1 or knockin mice expressing a CDK4 mutant insensitive to cyclin-dependent kinase inhibitors both exhibit $\beta$-cell hyperplasia (Marzo et al. 2004, Zhang et al. 2005), suggesting a linear relationship between these proteins and $\beta$-cell proliferation. Our results of PTTG inhibition of cell proliferation and PTTG-induced apoptosis shown in MIN6 cells in this report and previously in other cells (Yu et al. 2000a,b) demonstrate the requirement for an optimal concentration of PTTG that allows for normal $\beta$-cell proliferation. Although the ideal intracellular dose of PTTG is not apparent, PTTG may produce $\beta$-cell hyperplasia under an appropriate promoter, as PTTG driven by a subunit promoter causes pituitary hyperplasia (Abbud et al. 2005).

In summary, we demonstrate in this report that $\mathrm{mPTTG}$ fulfills the two criteria of a securin in murine insulin-secreting cells by live-cell imaging and that MPTTG binds to separase by immunocoprecipitation. Murine PTTG thus likely functions as a securin in murine insulin-secreting cells. PTTG overexpression is detrimental to cell proliferation and causes apoptosis in these cells.

\section{Acknowledgements}

Supported by NIH grants DK071870 (RY) and DK064169 (SM). The authors declare that there is no conflict of interest that would prejudice the impartiality of this scientific work.

\section{References}

Abbud RA, Takumi I, Barker EM, Ren SG, Chen DY, Wawrowsky K \& Melmed S 2005 Early multipotential pituitary focal hyperplasia in the alpha-subunit of glycoprotein hormone-driven pituitary tumor-transforming gene transgenic mice. Molecular Endocrinology 19 1383-1391.

Akino K, Akita S, Mizuguchi T, Takumi I, Yu R, Wang XY, Rozga J, Demetriou AA, Melmed S, Ohtsuru A et al. 2005 A novel molecular marker of pituitary tumor transforming gene involved in a rat liver regeneration. Journal of Surgical Research 129 142-146.

Ashizawa S, Brunicardi FC \& Wang XP 2004 PDX-1 and the pancreas. Pancreas 28 109-120.

Boelaert K, Yu R, Tannahill LA, Stratford AL, Khanim FL, Eggo MC, Moore JS, Young LS, Gittoes NJ, Franklyn JA et al. 2004 PTTG's C-terminal PXXP motifs modulate critical cellular processes in vitro. Journal of Molecular Endocrinology 33 663-677.

Bonner-Weir S 2000 Postnatal pancreatic beta cell growth. Endocrinology 141 1926-1929.

Brandeis M, Rosewell I, Carrington M, Crompton T, Jacobs MA, Kirk J, Gannon J \& Hunt T 1998 Cyclin B2-null mice develop normally and are fertile whereas cyclin B1-null mice die in utero. PNAS 95 4344-4349.

Chestukhin A \& DeCaprio JA 2003 Western blot screening for monoclonal antibodies against human separase. Journal of Immunological Methods $\mathbf{2 7 4}$ 105-113.

Fraenkel M, Caloyeras J, Ren S-G \& Melmed S 2006 Sex-steroid milieu determines diabetes rescue in pttg-null mice. Journal of Endocrinology 189 $519-528$.

Georgia S \& Bhushan A $2004 \beta$ cell replication is the primary mechanism for maintaining postnatal $\beta$ cell mass. Journal of Clinical Investigation 114 963-968. 
Ishikawa H, Heaney AP, Yu R, Horwitz GA \& Melmed S 2001 Human pituitary tumor-transforming gene induces angiogenesis. Journal of Clinical Endocrinology and Metabolism 86 867-874.

Jager H, Herzig A, Lehner CF \& Heidmann S 2001 Drosophila separase is required for sister chromatid separation and binds to PIM and THR. Genes \& Development 15 2572-2584.

Kushner JA, Ciemerych MA, Sicinska E, Wartschow LM, Teta M, Long SY, Sicinski P \& White MF 2005 Cyclins D2 and D1 are essential for postnatal pancreatic $\beta$-cell growth. Molecular and Cellular Biology 25 3752-3762.

Marzo N, Mora C, Fabregat ME, Martin J, Usac EF, Franco C, Barbacid M \& Gomis R 2004 Pancreatic islets from cyclin-dependent kinase 4/R24C (Cdk4) knockin mice have significantly increased b cell mass and are physiologically functional, indicating that Cdk4 is a potential target for pancreatic b cell mass regeneration in type 1 diabetes. Diabetologia 47 686-694.

Mei J, Huang X \& Zhang P 2001 Securin is not required for cellular viability, but is required for normal growth of mouse embryonic fibroblasts. Current Biology 11 1197-1201.

Nasmyth K 2002 Segregating sister genomes: the molecular biology of chromosome separation. Science 297 559-565.

Rane SG, Dubus P, Mettus RV, Galbreath EJ, Boden G, Reddy EP \& Barbacid M 1999 Loss of Cdk4 expression causes insulin-deficient diabetes and Cdk4 activation results in b-islet cell hyperplasia. Nature Genetics 22 44-52.

Wang Z \& Melmed S 2000 Characterization of the murine pituitary tumor transforming gene (PTTG) and its promoter. Endocrinology 141 763-771.

Wang Z, Yu R \& Melmed S 2001 Mice lacking pituitary tumor transforming gene show testicular and splenic hypoplasia, thymic hyperplasia, thrombocytopenia, aberrant cell cycle progression, and premature centromere division. Molecular Endocrinology 15 1870-1879.

Wang Z, Moro E, Kovacs K, Yu R \& Melmed S 2003 Pituitary tumor transforming gene-null male mice exhibit impaired pancreatic $\beta$ cell proliferation and diabetes. PNAS $1003428-3432$.
Yu R \& Melmed S 2001 Oncogene activation in pituitary tumors. Brain Pathology 11 328-334.

Yu R \& Melmed S 2004 Pituitary tumor transforming gene: an update. Frontiers of Hormone Research 32 175-185.

Yu R, Heaney AP, Lu W, Chen J \& Melmed S 2000a Pituitary tumor transforming gene causes aneuploidy and p53-dependent and p53independent apoptosis. Journal of Biological Chemistry 275 36502-36505.

Yu R, Ren SG, Horwitz GA, Wang Z \& Melmed S $2000 b$ Pituitary tumor transforming gene (PTTG) regulates placental JEG-3 cell division and survival: evidence from live cell imaging. Molecular Endocrinology 14 1137-1146.

Yu R, Lu W, Chen J, McCabe CJ \& Melmed S 2003 Overexpressed pituitary tumor-transforming gene causes aneuploidy in live human cells. Endocrinology 144 4991-4998.

Zhang X, Horwitz GA, Prezant TR, Valentini A, Nakashima M, Bronstein MD \& Melmed S 1999 Structure, expression, and function of human pituitary tumor-transforming gene (PTTG). Molecular Endocrinology 13 156-166.

Zhang X, Gaspard JP, Mizukami Y, Li J, Graeme-Cook F \& Chung DC 2005 Overexpression of cyclin D1 in pancreatic $\beta$-cells in vivo results in islet hyperplasia without hypoglycemia. Diabetes 54 712-719.

Zou H, McGarry TJ, Bernal T \& Kirschner MW 1999 Identification of a vertebrate sister-chromatid separation inhibitor involved in transformation and tumorigenesis. Science 285 418-422.

Received in final form 19 July 2006

Accepted 24 July 2006

Made available online as an Accepted Preprint 21 August 2006 